\title{
Acquired uterine arteriovenous malformation - a diagnostic dilemma
}

\author{
Agata Szpera-Goździewicz, Karolina Gruca-Stryjak, Grzegorz H. Bręborowicz, \\ Mariola Ropacka-Lesiak \\ Department of Perinatology and Gynecology, Poznan University of Medical Sciences, Poznan, Poland
}

Arteriovenous malformations (AVMs) are rare but considered as potentially life-threatening due to profuse or irregular bleeding from abnormal vascular connections. Acquired AVMs are predominantly related to various forms of iatrogenic uterine trauma.

A 34-year-old woman was admitted with a history of three episodes of heavy vaginal bleeding. Despite of these menstrual periods, the rest were normal. In 2011, the patient had undergone laparoscopy due to infertility and it revealed $3^{\text {rd }}$ stage of endometriosis according to Revised American Society of Reproductive Medicine classification. She also underwent simultaneous hysteroscopy with Dilatation and Curettage (D\&C). A few months later, she received in vitro fertilization and delivered spontaneously a healthy baby at term, with D\&C after delivery because of retained parts of placenta. In 2014, a heavy vaginal bleeding occurred and uterine AVM was suspected based on a transvaginal ultrasound examination. At the beginning of 2015, she underwent laparoscopy with ovarian cyst enucleation. Three weeks after the laparoscopy, she was admitted to the hospital with extremely heavy vaginal bleeding requiring red blood cell transfusion. The patient underwent a computed tomography (CT) scan with contrast, which confirmed AVM. She underwent transcatheter embolization of the right uterine artery and was prescribed combined oral contraceptive pills to avoid having a menstrual bleeding. Four weeks later, she was admitted to the hospital because of subsequent heavy vaginal bleeding; she again required red blood cell transfusion. In July 2015, the patient was admitted to our department. Transvaginal ultrasound showed a bulky uterus measuring $8.19 \times 7.74 \mathrm{~cm}$. On the grey image, a hypoechogenic lesion $66 \mathrm{~mm}$ in diameter was observed in the posterior wall of the uterus (Fig. $1 \mathrm{~A}, \mathrm{C}$ ). A colour Doppler ultrasound revealed a region of excessive

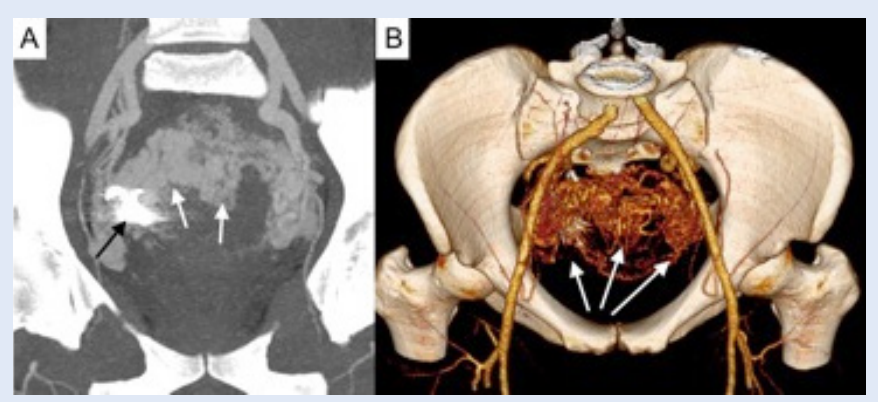

Figure 2. A. Maximum Intensity Projection (MIP) reconstruction. Coronal projection of computed tomography angiography showing tortuous vascular channels within uterus (arteriovenous malformation; white arrows) with arterial feeders from bilateral internal iliac arteries. On the right side the metal embolization coil is visible (black arrow). B. Computed tomography angiography $-3 \mathrm{D}$ reconstruction. The net of pathological vessels (white arrows) 
vascularisation in the posterior wall of the uterus (Fig. 1 B, D). Spectral Doppler ultrasound showed a PSV of $0.58 \mathrm{~m} / \mathrm{s}$ and RI of 0.36 . A CT angiography was required to assess the location of coils after uterine artery embolization. This exam confirmed the AVM, enlarged uterine arteries, and the presence of metal coils near the right side of the uterus (Fig. 2 A, B). The patient was advised to undergo embolization of the left uterine artery, however, she refused it. The woman insisted on a definitive procedure and desired hysterectomy because of fear of subsequent haemorrhage and no desire to preserve fertility. The histopathology showed AVM in the posterior wall of the uterus.

Unexplained uterine bleeding should be always an indication for colour Doppler ultrasonography. Definitely, three-dimensional CT angiography helps in confirming the diagnosis. 